\title{
Stadt, Bund und Reich in der Zollorganisation des kaiserzeitlichen Lykien. Eine neue Interpretation der Zollinschrift von Kaunos*
}

\section{Christian Marek}

„Der einzige Bereich, in dem fast jeder Provinzbewohner - ob peregrinen Rechts, latinischer oder römischer Bürger - kontinuierlich von der römischen Verwaltung aktiv betroffen wurde, war die Steuererhebung."1 Dieser Satz von Werner Eck läßt sich ohne weiteres in die moderne Staatenwelt übertragen, wo Steuererhebung jedermann ebenso kontinuierlich und aktiv betrifft, wie - so könnte man hinzufügen - Steuersenkung Regenten beim Volk beliebt macht. Auf Steigerung seiner Beliebtheit reichsweit hatte es denn auch schon im Jahre $58 \mathrm{n}$. Chr. der römische Kaiser Nero abgesehen, als er aufgrund sich häufender Beschwerden gegen die Zollpächter erwog, ob man nicht sämtliche Zölle überall einfach abschaffen solle. Die entsetzten Senatoren hatten Mühe, ihm das auszureden: Zwar zeuge sein Vorhaben von magnitudo animi, doch die realen Konsequenzen eines solchen Geschenks an die Menschheit kämen einer dissolutio imperii gleich, denn wenn erst einmal die Zölle aufgehoben seien, werde bald auch die Aufhebung der Tribute verlangt werden. Schließlich einigte man sich wenigstens auf die Beseitigung von Mißständen und auf Sonderregelungen. Das kaiserliche Edikt befahl, daß die

* Für die ehrenvolle Einladung, an der Marburger reunion von Schülern und Freunden zu Ehren Malcolm Erringtons teilnehmen zu dürfen, und für Hinweise zum Manuskript bedanke ich mich herzlich bei Hans-Ulrich Wiemer.

1 W. Eck, Der Kaiser, die Führungsschichten und die Administration des Reiches (von Vespasian bis zum Ende der antoninischen Dynastie), in: ders., Die Verwaltung des römischen Reiches in der Hohen Kaiserzeit, Bd. 2, Basel 1998, 3-145, hier: 132. 
diesbezügliche Gesetzeslage, bis dahin der Öffentlichkeit nicht zugänglich, künftighin publiziert werde. ${ }^{2}$

Zoll ist in der Antike eine Abgabe, die bei Überschreiten bestimmter Grenzen auf Handelswaren erhoben wurde. Zölle wurden an verschiedenen Kategorien von Grenzen erhoben, zuoberst an der Außengrenze des Imperium. Wir wissen von der Verzollung der über das Rote Meer importierten Güter des Ostafrika-, Arabien- und Indienhandels (Aromata, Gewürze, Perlen, Edelsteine, Seide etc.), daß in Alexandreia 25\% auf den Warenwert aufgeschlagen wurden. Die Zollgrenzen im Innern des Imperium orientierten sich an der Binnengliederung in Provinzen, waren aber nicht mit den Grenzen einzelner Provinzen identisch. Zollbezirke im Westen faßten mehrere Provinzen zusammen, und die Zöllner des portorium Asiae saßen u. a. am Bosporus. Provinzen waren wie Ägypten in Gaue oder wie Asia in Diözesen und Stadtterritorien unterteilt, und auch an diesen Grenzen wurden (sicher in Ägypten) Zölle erhoben. ${ }^{3}$ Was Art und Höhe des Zolls betrifft, so sind 2,5prozentige Wertzölle der gallischen und der spanischen Provinzen sowie Asiens bekannt.

Um die Organisation von Zöllen im römischen Weltreich auf regionaler bzw. lokaler Ebene zu ergründen, sind wir auf Textzeugnisse angewiesen, in denen zumeist von Sonderregelungen, Privilegien, Zusätzen oder Modifikati-

2 Tac. Ann. 13, 50-51: Eodem anno crebris populi flagitationibus immodestiam publicanorum arguentis dubitavit Nero an cuncta vectigalia omitti iuberet idque pulcberrimum donum generi mortalium daret. sed impetum eius, multum prius laudata magnitudine animi, attinuere senatores, dissolutionem imperii docendo, si fructus quibus res publica sustineretur deminuerentur: quippe sublatis portoriis sequens ut tributorum abolitio expostularetur. plerasque vectigalium societates a consulibus et tribunis plebei constitutas acri etiam tum populi Romani libertate; reliqua mox ita provisa ut ratio quaestuum et necessitas erogationum inter se congrueret. temperandas plane publicanorum cupidines, ne per tot annos sine querela tolerata novis acerbitatibus ad invidiam verterent. ergo edixit princeps ut leges cuiusque publici, occultae ad id tempus, proscriberentur; omissas petitiones non ultra annum resumerent; Romae praetor, per provincias qui pro praetore aut consule essent iura adversus publicanos extra ordinem redderent; militibus immunitas servaretur, nisi in iis quae veno exercerent; aliaque admodum aequa quae brevi servata dein frustra babita sunt. manet tamen abolitio quadragesimae quinquagesimaeque et quae alia exactionibus inlicitis nomina publicani invenerant. temperata apud transmarinas provincias frumenti subvectio, et ne censibus negotiatorum naves adscriberentur tributumque pro illis penderent constitutum.

3 Vgl. H.-J. Drexhage, Einflüsse des Zollwesens auf den Warenverkehr im römischen Reich. Handelshemmend oder handelsfördernd?, MBAH 13, 2, 1994, 1-15; H.-J. Drexhage/H. Konen/K. Ruffing, Die Wirtschaft des römischen Reiches. Eine Einführung, Berlin 2002, 145ff., 268f. mit Literaturangaben. Von den älteren Standardwerken sind hervorzuheben: M. Rostovtzeff, Geschichte der Staatspacht in der römischen Kaiserzeit, Leipzig 1904, bes. 363ff.; S. J. de Laet, Portorium, Étude sur l'organisation douanière chez les Romains, surtout à l'époque du Haut-Empire, Brügge 1949; F. Vittinghoff, Portorium, RE XXII, 1953, 346-399, bes. 384ff. 
onen die Rede und nur jeweils ein begrenztes Segment des zugrundeliegenden Systems zu erkennen ist, während das System als ganzes weitgehend im Verborgenen bleibt. Von vornherein ist mit erheblichen Unterschieden in den über 40 Provinzen zu rechnen, was deren vorrömische Tradition, Art und Umfang administrativer Strukturen in älterer Zeit betrifft. Im Orient blickte die Zollerhebung durch staatliche Autoritäten gewiß vielerorts auf eine sehr viel längere Geschichte und ein differenzierteres Regelwerk zurück als in manchen Regionen des Westens. Bei der Methode der Erhebung ist allgemein zwischen zwei Systemen, Pacht und Regie, zu unterscheiden. In der römischen Geschichte vollzieht sich von der Zeit der Republik bis in die mittlere Kaiserzeit ein, allerdings nicht gleichmäßiger und überall eintretender, Wandel: Das System der Pacht weicht allmählich dem System der Regie, an die Stelle großer privater Pachtgesellschaften tritt zunehmend die Erhebung durch staatliche Organe und subalternes Personal. Dieser Übergang ist etwa in der Zeit der Antonine vollzogen, Ägypten, Syrien und Palästina indessen behalten eine Sonderstellung. ${ }^{4}$

Eine unserer wichtigsten Quellen für das Zollwesen im römischen Reich sind Inschriften. Der Ortsname Turicum (Zürich) etwa findet seine früheste schriftliche Erwähnung als Name einer Zollstation auf dem Grabstein des Kindes eines Zöllners der statio Turicensis quadragesimae Galliarum, d. h. einer Zollstation zur Erhebung des 2,5-prozentigen Zolls auf den Warenwert bei Überschreiten der Grenze der gallischen Provinzen. ${ }^{5}$ Es gibt aber auch ausführlichere Texte, die Einblick in gesetzliche und organisatorische Aspekte bieten. Drei der umfangreichsten Zollinschriften aus dem Imperium, eine davon noch unveröffentlicht, gehen in die neronische Zeit zurück und verdanken ihre Entstehung offensichtlich der oben erwähnten Initiative des Kaisers. Sie stammen alle aus dem östlichen Teil des Reiches: Palmyra, Andriake und Ephesos. Die längste ist der 1881 gefundene Zolltarif von Palmyra. Eine $5 \mathrm{~m}$ breite beschriftete Wand südlich der Agora ist in über 400 Zeilen in griechischer und palmyrenischer Sprache beschriftet mit zwei Texten: erstens einem vom Stadtrat am 18. April $137 \mathrm{n}$. Chr. erlassenen Zollgesetz, zweitens einem

4 Für Ägypten vgl. P. J. Sijpesteijn, Customs Duties in Graeco-Roman Egypt (Studia Amstelodamensia ad epigraphicam, ius antiquum et papyrologicam pertinentia 17), Zutphen 1987.

5 CIL XIII $5244=$ ILS $1562=$ E. Howald/E.Meyer, Die römische Schweiz. Texte und Inschriften mit Übersetzung, Zürich 1940, Nr. 260. 
älteren Gesetz, das auf ein Edikt des neronischen Legaten C. Licinius Mucianus aus dem Jahr 68/9 n. Chr. zurückgeht. ${ }^{6}$

Das 1989 bekannt gewordene und seitdem sehr intensiv diskutierte Zollgesetz von Ephesos ${ }^{7}$ bezieht sich auf die gesamte Provinz Asia und spricht $u$. a. von Zollgrenzen, Anlage und Positionierung von Zollstationen, Sonderzöllen und Zollbefreiung, Deklarationspflicht und Konfiskationsrecht. Es handelt sich um ein Gesetz aus der Zeit der Republik, das durch Zusätze Zug um Zug ergänzt, $62 \mathrm{n}$. Chr. unter Nero in Rom archiviert und in einer griechischen Übersetzung in der Provinzhauptstadt Ephesos veröffentlicht wurde. Ohne Zweifel verdanken wir diese Inschrift der bei Tacitus bezeugten, durch das Edikt Neros verfügten Veröffentlichungspflicht, ut leges cuiusque publici, occultae ad id tempus, proscriberentur.

Ebenfalls in die Zeit Neros scheint eine in Andriake, neben dem hadrianischen Getreidespeicher kürzlich entdeckte, 87-zeilige Zollinschrift zu gehören, deren Edition Burak Takmer (Antalya) vorbereitet. Wir befinden uns am Fundort des Steins bei einem der wichtigsten Handelshäfen Lykiens, in einer unter Claudius neu eingerichteten Provinz des Imperium. Um die Zollorganisation dieser Provinz, Lycia, wird es in den folgenden Überlegungen gehen. Ausgangspunkt ist die neue Interpretation zweier längst bekannter Dokumente, von denen das eine aus unmittelbarer Nähe, aus Myra, das andere aus einer nicht weit entfernten Küstenstadt westlich davon, aus Kaunos, stammt.

6 CIS II 3, 3913; IGRR III 1056; OGIS 629; dazu K. Brodersen, Das Steuergesetz von Palmyra, in: E. M. Ruprechtsberger (Hg.), Palmyra - Geschichte, Kunst und Kultur der syrischen Oasenstadt (Linzer Archäologische Forschungen 16), Linz 1987, 153-162; M. Zahrnt, Zum Fiskalgesetz von Palmyra und zur Geschichte der Stadt in hadrianischer Zeit, ZPE 62, 1986, 279-293; J. F. Matthews, The Tax Law of Palmyra: Evidence for Economic History in a City of the Roman East, JRS 74, 1984, 157-180; J. Teixidor, Le tarif de Palmyre. Une commentaire de la version palmyrienne, Aula Orientalis 1, Barcelona 1983, 235-252.

7 Editio princeps: D. Knibbe/H. Engelmann, Das Zollgesetz der Provinz Asia, EA 14, 1989, 1-206. Aus der umfangreichen Literatur zu diesem Text seien hervorgehoben: M. Heil, Einige Bemerkungen zum Zollgesetz aus Ephesos, EA 17, 1991, 9-18; C. Nicolet, Le Monumentum Ephesenum et les dîmes d'Asie, BCH 115, 1991, 465-480; ders., Le Monumentum Ephesenum et la délimination du portorium d'Asie, MEFRA 105, 1993, 929-959; V. T. Spagnola, Lex portus Asiae. Un nuovo documento sull'appalto delle imposte, in: Atti del congresso della società italiana di storia del diritto sul tema „I rapporti contrattuali con la pubblica amministrazione nell'esperienza storica-giuridica“, Torino 17-19 ottobre 1994, Napoli 1996, 3-74; M. Dreher, Die Lex Portorii Asiae und der Zollbezirk Asia, EA 26, 1996, 111-127; ders., Das Monumentum Ephesenum und das römische Zollwesen, MBAH 16, 2, 1997, 79-95; C. Nicolet, Le Monumentum Ephesenum, la loi Terentia Cassia et les dîmes d'Asie, MEFRA 111, 1999, 191-215; G. D. Merola, Autonomia locale - governo imperiale. Fiscalità e amministrazione nelle province asiane, Bari 2001, bes. $203 \mathrm{ff}$. 
Man kann dieses Dokument als die Königin der Inschriften von Kaunos bezeichnen. Sie ist eingemeißelt an der Wand eines Brunnenhauses, das am Rande der Hafenagora aus den Trümmern wiederaufgerichtet wurde. ${ }^{8}$ Die Inschrift in mindestens drei Kolumnen (insgesamt 179 Zeilen) bedeckt heute die Stirnseite des rechten (südwestlichen) Antenvorsprungs auf vier Lagen (I-IV) und Teile der südwestlichen Außenwand von der obersten bis zur zweituntersten Lage mit insgesamt 24 Blöcken. Entdeckt wurden die beschrifteten Blöcke in den 50er Jahren des 20. Jahrhunderts von George E. Bean. Er hatte eine Rekonstruktion des schwierigen Textes vorgenommen, als die Blöcke noch nicht wieder an ihrem ursprünglichen Ort in der Mauer versetzt worden waren, was eine bewundernswerte Leistung darstellt. ${ }^{9}$ Seit Ende der 90er Jahre habe ich mehrere Kampagnen in Kaunos mit der gründlichen Neuaufnahme dieser Inschrift verbracht. Es stellte sich heraus, daß ganze Textpartien Bean unbekannt geblieben waren. Ich komme zu Ergebnissen, die das Verständnis des ganzen Dokumentes in einem erheblichen Maße verändern. Die wichtigsten Ergebnisse sollen im folgenden vorgestellt werden. ${ }^{10}$

Um auf die Problematik der Inschrift von Kaunos hinzuführen, nehme ich am besten von jener Zollinschrift von Myra meinen Ausgang, die $1966 \mathrm{im}$ Theater dieser lykischen Stadt entdeckt wurde. Es handelt sich um ein Platte von $80 \mathrm{~cm}$ Höhe, oben gebrochen; der erhaltene Teil ist, wie aus der Gruß-

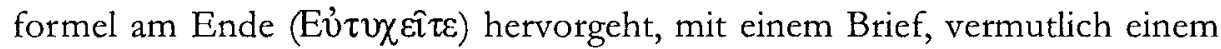
Statthalterbrief, beschriftet, der ziemlich sicher aus hadrianischer Zeit stammt. Er ist 1975 von Michael Wörrle mit ausführlichem Kommentar ediert worden: ${ }^{11}$

8 C. Ișik, Die Ausgrabungen in Kaunos bis zur Entdeckung der Bilingue, in: W. Blümel/P. Frei/Chr. Marek (Hgg.), Colloquium Caricum. Akten der Internationalen Tagung über die karisch-griechische Bilingue von Kaunos, 31. 10. - 1. 11. 1997 in Feusisberg bei Zürich, Berlin 1998, 183-202, hier: 192f.

9 G. E. Bean, Notes and Inscriptions from Caunus II, JHS 74, 1954, 85-110, hier: 97-99 Nr. 38 = SEG XIV 639.

10 Eine eingehende Behandlung der epigraphischen Problematik und ausführliche Interpretation des Textes wird im Rahmen einer neuen Edition erfolgen: C. Marek, Die Inschriften von Kaunos (Vestigia. Beiträge zur Alten Geschichte 55), München 2006, Nr. 35. Ich verweise hier auf die Abschnitts- und Zeilenzählung von Beans Text.

11 M. Wörrle, Zwei neue Inschriften aus Myra zur Verwaltung Lykiens in der Kaiserzeit, in: J. Borchardt (Hg.), Myra. Eine lykische Metropole in antiker und byzantinischer Zeit, Berlin 1975, 255-300, hier: 286-300 = SEG XXXIII 1147. 


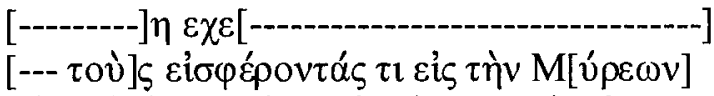

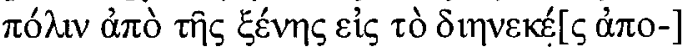

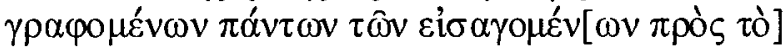

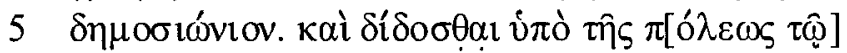

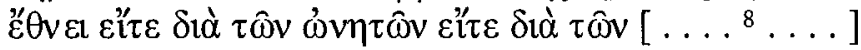

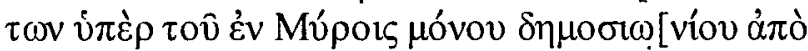

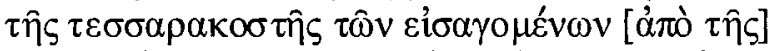

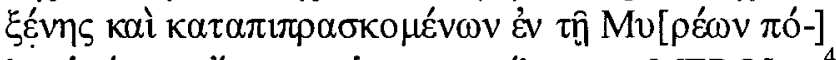

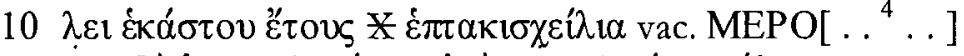

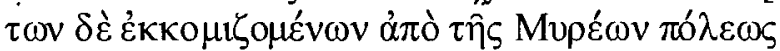

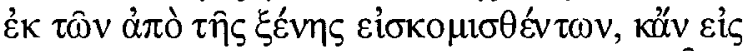

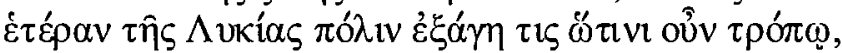

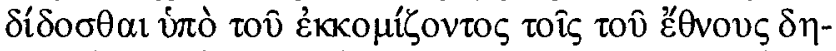

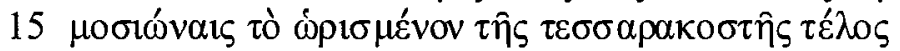
ì نं

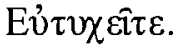

[-- sollen diejenigen], die etwas aus der Fremde in die Stadt Myra einführen, stets [--], nachdem alle Einfuhrgüter an das Demosionion schriftlich deklariert worden sind, (Zoll zahlen ? - oder: nach Deklaration an das Demosionion zahlen?). Und die Stadt soll dem Bund (Ethnos), sei es durch die Pächter, sei es durch die [--], allein für das Demosionion in Myra von der 2,5-prozentigen Abgabe (Tessarakoste) auf Einfuhrgüter aus der Fremde, die in der Stadt Myra verkauft werden, jedes Jahr 7000 Denare zahlen. [--] auf diejenigen Güter, die aus der Fremde eingeführt, aus der Stadt Myra aber wieder ausgeführt werden, auch wenn irgend jemand sie, wie auch immer, in eine andere Stadt Lykiens ausführt, soll der Exporteur den Pächtern des Bundes (Ethnos) den festgesetzten Zoll der 2,5-prozentigen Abgabe (Tessarakoste) zahlen oder der Beschlagnahme unterliegen, gemäß dem Zollgesetz (Demosionikos Nomos).

Gehabt Euch wohl!

Wörrle hat in diesem Text vier verschiedene Bestimmungen erkannt:

1) eine Deklarationspflicht,

2) einen Zoll unter städtischer Verwaltung (geht aus Z. 5 hervor): 2,5\% auf

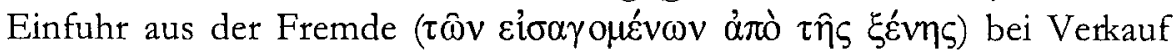

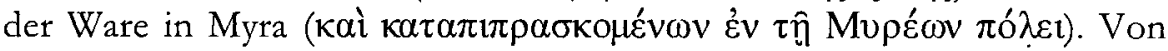


diesen Zolleinnahmen muß die Stadt jedes Jahr eine Pauschale von 7.000 Denaren an den Lykischen Bund abführen.

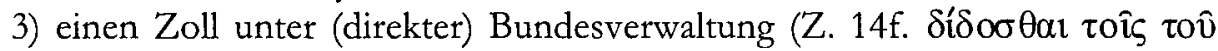

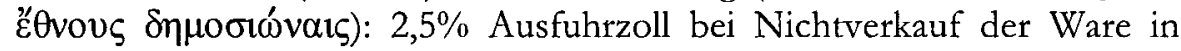
Myra und ihrer Wiederausfuhr, nicht nur, wenn die Ware Lykien wieder verläßt, sondern auch, wenn sie innerhalb Lykiens weitertransportiert wird

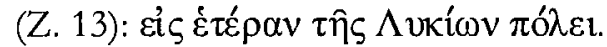

4) das Institut des commissum, d. h. das Recht des Zöllners, im Falle des Zollbetrugs selbsthilfeähnliche Zwangsmittel anzuwenden -, Konfiskation der Ware oder, wenn diese nicht greifbar war, Pfandnahme ${ }^{12}$ - kommt zur

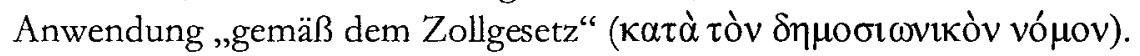

Gegen Wörrles Interpretation dieser Urkunde haben Helmut Engelmann 1985 und Hertha Schwarz 2001 die These von einer lykischen Zollunion aufgestellt. ${ }^{13}$ Auf Ware, die nach Lykien hereinkam, so die beiden Forscher, sei grundsätzlich nur einmal Zoll erhoben worden: Entweder bei Verkauf in der der Außengrenze des Zollbezirkes nächstgelegenen Stadt (in diesem Fall die Stadt Myra), unter städtischer Hoheit. Oder bei Wiederverlassen des Territoriums dieser Stadt an der Außengrenze - egal, in welche Richtung, sei es ins Innere Lykiens, sei es wieder aus dem lykischen Bezirk heraus - unter Bundeshoheit. Von da an habe man die Ware zollfrei in ganz Lykien bewegen können. Das Dokument, so macht Schwarz geltend, habe überhaupt nichts mit dem römischen portorium zu tun, sondern sei eine rein innerlykische Angelegenheit.

Zunächst einmal birgt die Theorie von der Zollunion Lykiens eine Konsequenz in sich, die Engelmann und Schwarz nicht ausgeführt haben: Nehmen wir an, ein Händler aus Alexandreia verkaufte nur die Hälfte seiner Ware in Myra. Die andere Hälfte mußte er wieder ausführen, zahlte Zoll und erhielt dafür eine Quittung. Er fuhr westwärts und führte die Restware in Patara ein. Die Stadt Patara durfte dann keinen Zoll erheben. Der Grund: Diese Ware war schon einmal in einer anderen lykischen Stadt angeboten und dort verzollt worden. Der Gemeinde konnten Zolleinnahmen nur dann zufließen, wenn Ware aus der Fremde direkt nach Patara kam. Völlig abge-

12 Dazu G. Klingenberg, Der Verfall nicht deklarierter Sachen im römischen Zollrecht, Graz 1977.

13 H. Engelmann, Die Zollinschrift von Myra, ZPE 59, 1985, 113-119; H. Schwarz, Soll oder Haben? Die Finanzwirtschaft kleinasiatischer Städte in der Römischen Kaiserzeit am Beispiel von Bithynien, Lykien und Ephesos (29 v. Chr. - 284 n. Chr.), Bonn 2001, 395ff.; H. Schwarz, Anmerkungen zu der Zollinschrift aus Myra, EA 33, 2001, 15-38. 
schnitten davon wären die binnenländischen Städte Lykiens gewesen. Der ganze Lykische Bund wäre zweigeteilt gewesen in Städte mit Zollprivileg (an der Außengrenze) und Städte ohne Zollprivileg (im Binnenland). Ob diese Konsequenz für ein Modell der Organisation des lykischen Zollwesens annehmbar ist, bleibt zumindest fraglich.

Des weiteren ist jedoch zu fragen, ob die Theorie externer Evidenz standhalten kann. Problematisch erscheint in dieser Beziehung das Beispiel von Oinoanda, einer Stadt im Binnenland, die gemäß der 1988 veröffentlichten Stiftungsurkunde des Demosthenes durchaus Zölle erhob. Darauf sind Wörrle und Schwarz mit je verschiedener Interpretation eingegangen. ${ }^{14}$ Entscheidendes zur Lösung des Problems trägt nun aber die Zollinschrift von Kaunos bei. Sie ist nie in diese Diskussion einbezogen worden, und das hat seinen Grund in dem seit Beans Bearbeitung vorherrschenden Verständnis des Dokumentes. In wesentlichen Punkten erweist sich dieses Verständnis als falsch:

Erstens datiert die Inschrift nicht in das 1. Jh. n. Chr., sondern ist hadrianisch wie die Inschrift aus Myra. Das ergibt die erstmalige Entzifferung ihres Anfangs. Zweitens - und das ist von größerer Tragweite - steht daselbst nach der Kaisertitulatur die Jahresdatierung nach einem Erzpriester des Lykischen Bundes geschrieben. Die Stadt Kaunos muß folglich zu dieser Zeit Mitglied des Bundes gewesen sein und mithin zur römischen Provinz Lycia gehört haben, d. h. zu derselben Provinz wie die Stadt Myra. Sie muß, wenn man von einer Organisation des römischen Zollwesens auf Provinzebene wie in Asia ausgeht, demselben Zollbezirk, nämlich dem lykischen, angehört und denselben gesetzlichen Bestimmungen unterlegen haben. Drittens aber handelt es sich bei der Inschrift von Kaunos nicht um ein städtisches Zollgesetz, wie bisher angenommen, sondern um ein ganzes Urkundendossier über eine private Stiftung zweier Kaunier. Der starke Textverlust auf der Ante und auf Teilen der Wand macht eine genaue Bestimmung der einzelnen Urkunden des Dossiers zwar unmöglich. Handelt es sich um Dekrete des Rates, der Volksversammlung und des Rates oder um Erlasse einer höheren Instanz? Die ausführlichen Zollregularien stehen aber jedenfalls in Zusammenhang mit dem Stiftungszweck.

Worin besteht dieser und was wird von diesen beiden Männern gestiftet?

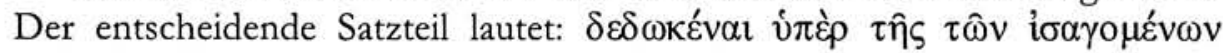

14 M. Wörrle, Stadt und Fest im kaiserzeitlichen Kleinasien. Studien zu einer agonistischen Inschrift aus Oinoanda (Vestigia. Beiträge zur Alten Geschichte 39), München 1988, 213f.; Schwarz, Finanzwirtschaft (wie Anm. 13), 369-371; dies., EA 332001 (wie Anm. 13), 34-35 Anm. 30. 


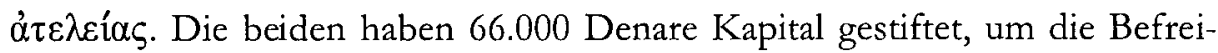
ung des Handels vom Einfuhrzoll zu finanzieren - eine private Subvention öffentlicher Einkünfte, einmalig bisher in unserer epigraphischen Dokumentation, wenn man von einer kurzen Inschrift aus Xanthos (TAM II 291) absieht. Dieser Stiftungszweck ist im Hinblick auf das wirtschaftliche Denken und Handeln in der Antike bemerkenswert. Stifter übernehmen häufig die Finanzierung von Leistungen, die für die Bürgerschaft erbracht werden, und entlasten damit den Haushalt ihrer Heimatstadt, gleichgültig, ob es sich um die Errichtung von Bauwerken, um die Bezahlung von Festen und Wettkämpfen oder um die Herstellung von Münzgeld handelt. Im vorliegenden Fall jedoch dient die Stiftung dem Zweck, die Stadt gegen das Risiko zu versichern, daß ihr durch eine Befreiung des Handels vom Einfuhrzoll ein Ausfall an Einnahmen entsteht. Hinter der Stiftung steht also der Wille, durch eine partielle Atelie den Import von Waren nach Kaunos zu fördern. Damit scheint eine gewisse Einschränkung der Aussage Moses I. Finleys notwendig zu sein, es habe in der Antike „nicht die geringste Vorstellung davon (gegeben), wie man die inländische Produktion schützen, lebensnotwendige Importe herbeiführen und eine Zahlungsbilanz pflegen könnte". ${ }^{15}$ Eine solche Vorstellung muß in Kaunos vorhanden gewesen sein, auch wenn sie auf ein einfaches Problem, die qualitative und quantitative Verbesserung und wohl auch Verbilligung des Warenangebotes, gerichtet war.

Auf den zweiten Blick indessen liefert das kaunische Beispiel auch eine Bestätigung der Gedanken, die Finley zum Thema „Der Staat und die Wirtschaft" (so ist das einschlägige Kapitel überschrieben) geäußert hat. Denn die in Kaunos eingeführte Atelie für Importwaren bedeutete lediglich eine punktuelle Ausnahme von einem in der ganzen Provinz gültigen Zollsystem, das, wie zu zeigen sein wird, Importe und Exporte im Prinzip völlig gleich behandelte; zudem wurde sie durch die Spendabilität privater Stifter abgesichert. Insofern scheint Finley durchaus Recht zu behalten, wenn er feststellt: „Niemals gibt es einen Hinweis darauf, daß die Befreiung von Hafenzöllen als ein (angemessener oder unangemessener) Beitrag zu der Konkurrenzfähigkeit des Empfängers in Handel oder Herstellung verstanden wurde; sie hatte dieselbe Bedeutung wie reservierte Plätze im Theater." 16

Ich skizziere im folgenden kurz den Aufbau des Textes, soweit er noch erkennbar ist: Nach der Stiftungsankündigung ist zunächst eine allgemeine Zollpflicht formuliert. Dann wird, zusammen mit den Namen der Stifter, die gestiftete Geldsumme und der Verwendungszweck genannt. Es folgen Be-

15 M. I. Finley, Die antike Wirtschaft, München 21984, 193.

16 Ebd. 
stimmungen, bei denen wiederholt Bezug genommen wird auf einen Demosionikos Nomos. Erst im folgenden Abschnitt wird die von der Stiftung getragene Zollbefreiung näher beschrieben, wobei vor allem deutlich wird, daß sie nur für Ware gilt, die zum Verkauf in Kaunos bestimmt ist:

„Alle, die etwas aus dem Ausland einführen, zu Wasser oder zu Lande, was es auch ist, ob es zum Eigenbedarf oder zum Verkauf hier bestimmt ist, unterliegen keinerlei Zahlungspflicht auf Hafenzoll, ${ }^{17}$ wenn es sich nicht um Ware handelt, die ausdrücklich als zollpflichtig definiert ist, wobei die Pächter des Hafenzolls weder Geld auf die Einfuhrgüter kassieren dürfen, wie es auf die jeweilige Warenklasse festgesetzt ist, noch von den eingeführten Gütern selbst etwas nehmen dürfen, sei es als Zoll, sei es im Namen einer Spende an Aphrodite, sei es als irgendeine sonstige Gebühr." “18

Verkaufswillige Händler erhalten einen zusätzlichen Anreiz: Ist ihre Ware zum Verkauf in Kaunos bestimmt, mithin zollfrei eingeführt worden, findet jedoch wider Erwarten keinen Absatz, so darf sie in angemessener Frist zollfrei wieder ausgeführt werden:

„Die Fremden, die zu Schiff ankommen und etwas verkaufen wollen, und die ebenfalls nach ihrer Ankunft das Privileg der Zollfreiheit auf Einfuhrgüter haben, unterliegen nicht dem Ausfuhrzoll oder irgendeiner als Spende an Aphrodite deklarierten Abgabe, soweit es sich um Ware handelt, die, eingeführt von ihnen und an Land gebracht, unverkauft bleibt, unter der Bedingung daß sie < selber> dieselbe Ware innerhalb von 20 Tagen wieder auf die Schiffe laden und ausführen. Und auch diejenigen Fremden sollen weder dem Ausfuhrzoll noch irgendeiner Spende an Aphrodite unterliegen, die etwas zum Verkauf auf dem Landweg hereinbringen, soweit es sich um Ware handelt, die sie nicht verkaufen können und die sie selbst auf demselben

17 Der Begriff $\dot{\varepsilon} \lambda \lambda_{\imath} \mu \varepsilon ́$ viov steht in Kaunos für die Gesamtheit der Zolleinnahmen auf den Warenumschlag zu Wasser und zu Lande (!), und nicht für eine Hafengebühr. Die ausführliche Beweisführung für diese Interpretation wird im Corpus der Inschriften von Kaunos (siehe Anm. 10) erfolgen.

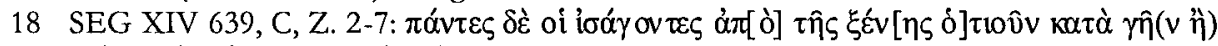

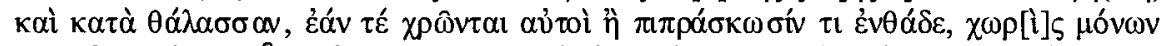

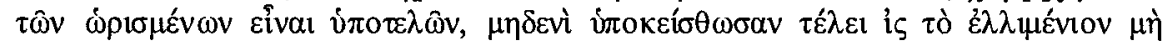

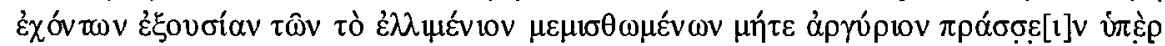

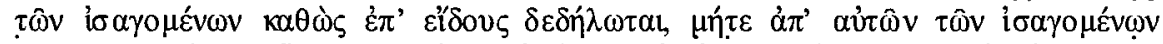

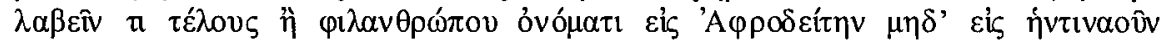

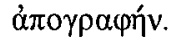




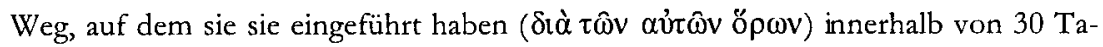
gen ausführen.“19

Aus der Interpretation dieses Abschnitts und anderer Textstellen treten auffällige Beziehungen zu der Zollurkunde aus Myra zutage, auf die ich im folgenden nacheinander eingehe:

\section{Demosionikos Nomos}

Bean hat (1954) diesen Nomos für ein städtisches, nur in Kaunos gültiges Zollgesetz angesehen, und die Forschung ist ihm darin gefolgt. Niemand hat nach der Publikation der Myra-Inschrift (1975), wo derselbe Begriff Demosionikos Nomos, vorkommt, darauf hingewiesen, daß ein solcher Nomos in zwei nicht weit voneinander entfernten Städten, in Kaunos wie in Myra, existierte. $\mathrm{Da}$ Kaunos, wie wir jetzt wissen, zum Zeitpunkt der Stiftung zum Lykischen Bund gehört, kann nicht von zwei verschiedenen Gesetzen die Rede sein, einem Bundesgesetz in Myra und einem städtischen Gesetz in Kaunos. Es ist nahezu zwingend, daß damit auf ein Zollgesetz für ganz Lykien verwiesen wird.

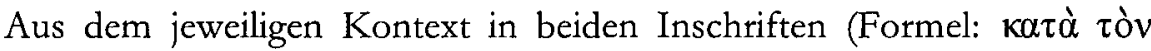

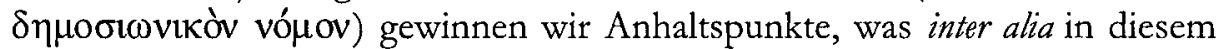
Gesetz stand. In Kaunos definierte es bestimmte Waren, die in jedem Fall zollpflichtig blieben, also der durch die Stiftung ermöglichten Befreiung vom

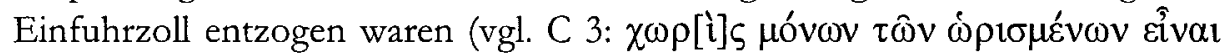

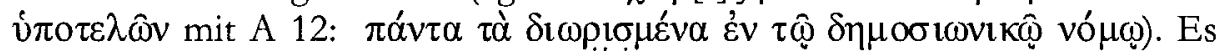
regelte in Kaunos wie in Myra die Beschlagnahme durch die Zöllner (commissum). Es regelte in Kaunos (B 9f.) die grundsätzliche Befreiung von der Zollpflicht bei Ware für den Eigenbedarf und bestimmte einen Sondertarif bei Ausfuhr von Sklaven. Für nahezu jede dieser gesetzlichen Bestimmungen liegen eindeutige Parallelen aus dem römischen Zollgesetz von Ephesos

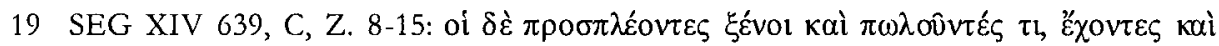

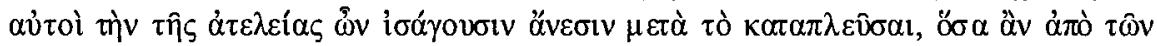

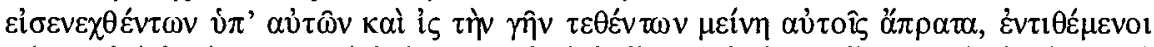

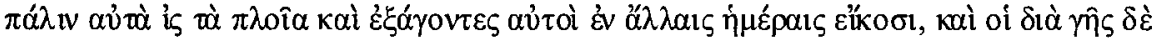

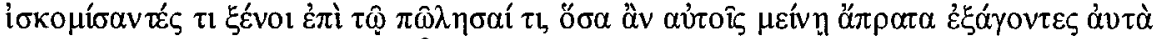

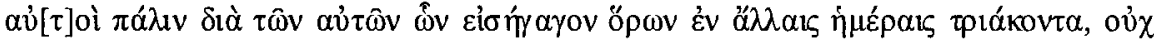

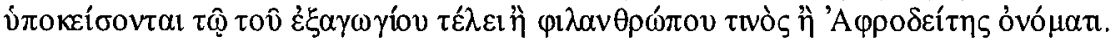


vor, ${ }^{20}$ auch dies ein klares Indiz, daß wir es hier mit nichts anderem zu tun haben als einem von den Römern erlassenen Zollgesetz der Provinz Lycia, dem Gegenstück des Zollgesetzes von Asia.

\section{Zollhoheit}

Aus dem Text in Kaunos geht klar hervor: Außer den im Zollgesetz definierten Waren unterliegt der Zollpflicht auch weiterhin grundsätzlich der gesam-

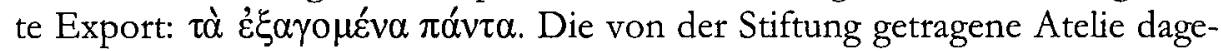
gen betrifft ausschließlich den Importzoll auf die zum Verkauf in Kaunos bestimmte Ware (und deren Wiederausfuhr innerhalb von Fristen). Das ist eine sehr auffällige Parallele zu der Tatsache, daß es in Myra eine Hoheit der Stadt nur über Importzoll gab, nicht dagegen über Exportzoll, und über Importzoll auch nur dann, wenn die Ware in der Stadt verkauft wurde. Man kann auf Grund dieser Parallele und der Tatsache der Zugehörigkeit zu demselben Zollbezirk vermuten, daß wie Myra auch Kaunos die Hoheit nur über Zölle auf Einfuhren besaß unter exakt derselben Bedingung, sodaß wohl auch die Kaunier aus den Zolleinnahmen von am Ort verkaufter Ware eine Jahrespauschale an den Bund abführen mußten. Die private Stiftung dürfte dann so bemessen gewesen sein, daß aus den Zinseinnahmen die Zahlungsverpflichtung gegenüber dem Bund auf jeden Fall eingelöst werden konnte.

\section{Ausfuhrzoll}

Das Territorium von Kaunos bildet sowohl an der Land- wie an der Seeseite die Außengrenze des Zollbezirkes Lykien. Aus der oben zitierten Passage (C) geht hervor, daß die zollfreie Wiederausfuhr nicht verkaufter Ware in bestimmter Frist beschränkt ist auf den Ort, wo die Ware eingeführt wurde. Bei einer Zollunion Lykiens, wie Engelmann und Schwarz sie vertreten, hätte der fällige Zoll bei Ausfuhr an jeder Stelle erhoben werden können (und müssen). Warum also hier der Zwang zur Wiederausfuhr am Ort der Einfuhr? Der Sinn dieser Bestimmung ist es, die zum Verkauf in Kaunos angemeldete, dann aber dort nicht verkaufte Ware von einfacher Transitware zu unterscheiden. Das aber impliziert, daß bei Warenausfuhr an anderer als an der Einfuhrstelle ein Zoll fällig wurde, der vom Importzoll verschieden war. Fol-

20 Konfiskation: $\iint 19,38$; Eigenbedarf: $\int \S 35,37$ (vgl. $₫ 25-27$ für Staat und Militär); Sklaven: $\iint 3,41,51$. 
gerichtig lesen wir an mehreren Stellen (in C 14, D 11, E 9.14, F 6-7.9) von

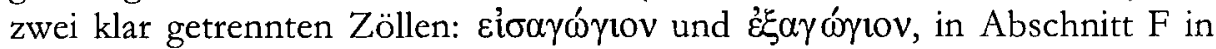

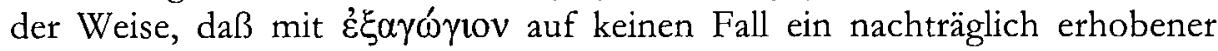
Einfuhrzoll gemeint sein kann. Unter der Voraussetzung, daß Kaunos zum Lykischen Bund gehörte, dürfte damit die Theorie von der Zollunion Lykiens in hadrianischer Zeit als gescheitert gelten.

\section{Zollhöhe}

Über die Zollhöhe erfahren wir Grundsätzliches an zwei Stellen der Inschrift aus Kaunos: In A 12 ist im Zusammenhang mit der Zollpflicht von einem Wertzoll in Höhe von 5\% die Rede. In C 5f, werden den Zöllnern - auf Grund der Atelie - zwei Arten der Zollerhebung verboten:

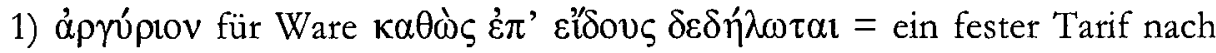
Warenklasse; diese Regelung dürfte etwa auf Sklaven zutreffen. Es muß ein Katalog dieser Waren mit Angaben der Zollhöhe existiert haben.

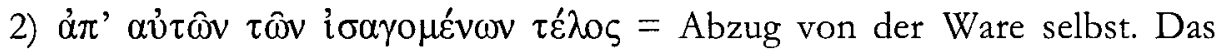
kommt auf einen prozentualen Wertzoll hinaus.

Für die zweite Art der Verzollung zeigt sich wiederum eine auffällige Beziehung zum Zolldokument von Myra. Der fünfprozentigen Abgabe (Eikoste) in Kaunos steht dort eine 2,5-prozentige (Tessarakoste) gegenüber, genau die Hälfte. Wenn man nicht annehmen will, daß Händler in Kaunos normalerweise den doppelten Zoll zu zahlen hatten wie in einer Hafenstadt desselben Zollbezirks weiter östlich, dann drängt sich folgende Vermutung auf: Der Lykische Bund erhob bei jeder Grenzüberschreitung 2,5\%, die doppelte Verzollung ergibt also 5\%. Sehen wir uns die Bestimmung in der Myra-Inschrift näher an. Der in Zeilen 10-15 gemeinte Ausfuhrzoll, die Tessarakoste, wäre

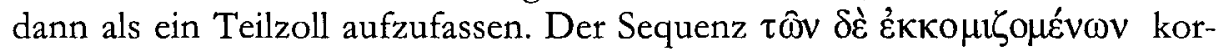
respondierte im verlorenen, oberen Teil der Inschrift offensichtlich ein mit

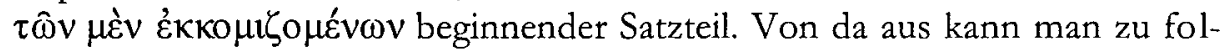
gender Überlegung gelangen: Es handelt sich bei dieser Urkunde, wie aus der Grußformel am Schluß deutlich wird, um einen Kaiser- oder, wahrscheinlicher, einen Statthalterbrief, dessen Ausführungen - wie üblich - die Entscheidung in einem Streitfall herbeiführen. Sehr wichtig ist nun die in der ersten Zeile gerade noch erhaltene Buchstabensequenz, die man m. E. kaum

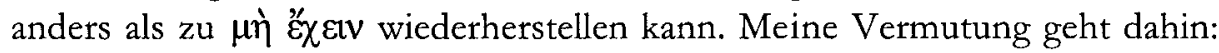


Dem Bundeszoll war infolge einer von der Stadt Myra gewährten Atelie ein Einnahmeausfall entstanden. Vielleicht hatte der Bund daraufhin versucht, die Hoheit über die Zölle ganz an sich zu ziehen. Jedenfalls ging eine Klage, von welcher Partei auch immer, an den Statthalter. Der Statthalter vertbot die

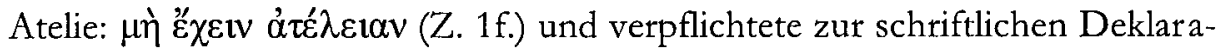
tion. Zugleich aber beschränkte er die Zollhoheit des Bundes auf Transit bzw. Export und bestätigte der Stadt ihre Hoheit über den Zoll auf am Ort verkaufte Ware.

Damit wäre aus der kombinierten Interpretation der beiden Dokumente aus Kaunos und Myra die kaiserzeitliche Zollorganisation Lykiens in den Grundzügen sichtbar: Überschritt eine von außen in diesen Bezirk hereingebrachte Ware die Grenze, so gab es zwei Möglichkeiten:

1) Die Ware wurde in der Hafenstadt oder einer Stadt an der Außengrenze auf der Landseite verkauft. Dann durfte diese Stadt Zoll einnehmen. Kaunos durfte darauf (und nur darauf) Atelie gewähren. Die Stadt mußte aus diesen Einnahmen jedoch eine Jahrespauschale an den Bund abführen, und wenigstens diese Summe mußte in Kaunos aus der Stiftung aufzubringen sein.

2) Wurde (von den Sondervergünstigungen in Kaunos abgesehen) Ware wieder ausgeführt, erhob der Bund durch seine Pächter Zoll, und zwar doppelt, Ein- und Ausfuhrzoll, insgesamt $5 \%$.

Hieraus ergibt sich für die Finanzorganisation des Lykischen Bundes die Schlußfolgerung, daß er zwei getrennte Kassen mit Zolleinnahmen füllte: Erstens eine Zollkasse aus den Einkünften seiner Zöllner, zweitens eine Zollkasse aus den Einkünften der städtischen Pauschalzahlungen. Rom brauchte den lykischen Zoll bloß noch aus den beiden Bundeskassen abzuschöpfen.

Die gewohnte Zuordnung von Kaunos zur kaiserzeitlichen Provinz Asia hätte uns eigentlich schon nach der Veröffentlichung des Monumentum Ephesenum verdächtig erscheinen können. Denn die Zollinschrift von Kaunos paßt nicht mit dem zusammen, was in dem Zollgesetz über die Organisation des asiatischen Zollbezirks zu lesen ist. Hier gab es zwischen Bosporus und Pamphylien einen Ring von über 20 Küstenstädten, in denen die Pächter des römischen portorium die Ein- und Ausfuhr von Waren besteuerten. Über eine Zollhoheit einzelner Städte ist nichts bekannt. Eine Befreiung der Händler vom Einfuhrzoll in einem oder mehreren dieser Häfen wäre mit diesem System unvereinbar. Die Annahme hingegen, daß in Kaunos neben dieser römischen Zollverwaltung noch eine städtische existiert habe, daß die Zollbe- 
freiung sich einzig auf dieses städtische Zollsystem beziehe und ein nur in dieser Stadt gültiges, weil von ihr erlassenes Gesetz vorliege, bedeutete eine unerhörte fiskalische Ausnahme für eine Hafenstadt an der Außengrenze des Zollbezirkes. Es gibt keinen Hinweis darauf, daß Kaunos ein solches Privileg besessen haben könnte, und der erhaltene Text enthält nicht die geringste Andeutung einer Koexistenz einer römischen mit einer städtischen Zollverwaltung. Das Problem besteht nicht länger, seit wir wissen, daß Kaunos zur Zeit der Abfassung des Zolldokumentes der Provinz Lycia angehörte. Mehr noch: Jetzt wird deutlich, daß zwei lykische Städte sich auf dasselbe Zollgesetz beziehen, und daß die Zollverwaltung in beiden Städten im Prinzip gleich organisiert war. Eben mit dem in diesen beiden Zollreglementen zitierten Demosionikos Nomos fassen wir nichts anderes als die in Form eines Gesetzes von römischen Autoritäten verfügte Grundlage der Organisation der Zölle in ganz Lykien, in welchem u. a. die Verzahnung der Zollerhebung durch die Städte und durch den Bund geregelt war.

Diese Zollorganisation ersparte es dem römischen Fiskus, das lykische portorium durch eigene publicani oder procuratores an allen Außengrenzen erheben zu müssen; er brauchte bloß die Zahlungen entgegenzunehmen, die der lykische Bund an ihn abführte. Das kaiserzeitliche Zollwesen Lykiens war mithin völlig anders konstruiert als das in Asien. Die Verwaltung der Einfuhrzölle übte jede Stadt für sich aus, und diese Einnahmen flossen auch in ihre Kasse, wenn die Ware am Ort verkauft wurde. Dafür entrichtete jede Stadt eine feste Zollsteuer an den Bund. Bundesangelegenheit dagegen blieb die Zollerhebung auf grenzüberschreitende Ware an jeder Außen- und Binnengrenze, solange die Ware nicht verkauft wurde. Wahrscheinlich wurde dieses System bereits vom Städtebund geschaffen und mit in die Provinz unter Claudius eingebracht. ${ }^{21}$ Die Römer haben es im wesentlichen so belassen und - vielleicht wiederum unter Nero - eine schriftliche Fixierung (und Veröffentlichung?) geltender gesetzlicher Grundlagen und Rahmenordnungen (Zollhöhe, Zollbefreiung bei Eigenbedarf, Beschlagnahmerecht der Zollpächter) verfügt. Wieder einmal zeigt sich, wie wenig Rom geneigt war, historisch gewachsene Systeme umzukrempeln, dies umso weniger, wenn sie zum Nutzen der Herrscher funktionierten.

21 Zur Problematik der Provinzialisierung vgl. H. Brandt/F. Kolb, Lycia et Pamphylia. Eine römische Provinz im Südwesten Kleinasiens, Mainz 2005, $21 \mathrm{ff}$. 
